\title{
A functional genetic variant in fragile-site gene FATS modulates the risk of breast cancer in triparous women
}

\author{
Fangfang Song ${ }^{1,4 \dagger}$, Jun Zhang ${ }^{2,3 \dagger}$, Li Qiu ${ }^{2,4 \dagger}$, Yawen Zhao 2,4 , Pan Xing ${ }^{2,4}$, Jiachun Lu ${ }^{5^{*}}$, Kexin Chen ${ }^{1,4^{*}}$ \\ and Zheng $\mathrm{Li}^{2,4^{*}}$
}

\begin{abstract}
Background: The fragile-site associated tumor suppressor (FATS, formerly known as C10orf90), a regulator of p53-p21 pathway has been involved in the onset of breast cancer. Recent data support the idea that the crosstalk between FATS and p53 may be of physiological importance for reproduction during evolution. The aim of the current study was to test the hypothesis that FATS genetic polymorphism can influence the risk of breast cancer.

Methods: We conducted population-based studies in two independent cohorts comprising 1532 cases and 1573 controls in Tianjin of North China, and 804 cases and 835 controls in Guangzhou of South China, coupled with functional validation methods, to investigate the role of FATS genetic variant in breast cancer risk.

Results: We identified a functional variant rs 11245007 (905C > T, 262D/N) in fragile-site gene FATS that modulates p53 activation. FATS-262 N exhibited stronger E3 activity to polyubiquitinate p53 than did FATS-262D, leading to the stronger transcriptional activity of p53 and more pronounced stabilization of p53 protein and its activation in response to DNA damage. Case-control studies found that $C T$ or $\Pi$ genotype was significantly associated with a protective effect on breast cancer risk in women with parity $\geq 3$, which was not affected by family history.

Conclusions: Our findings suggest the role of FATS-p53 signaling cascade in suppressing pregnancy-related carcinogenesis and potential application of FATS genotyping in breast cancer prevention.
\end{abstract}

Keywords: Breast cancer, FATS, Single-nucleotide polymorphism, p53, Parity

\section{Background}

Breast cancer is both the most common malignancy and the one causing the highest number of cancer deaths in women worldwide. For most sporadic breast cancers, it has been suggested that genetic polymorphisms, especially single nucleotide polymorphisms (SNPs) in low-penetrance susceptibility genes in concert with environmental exposures may be more important. The p53 tumor suppressor protein, through its downstream target $\mathrm{p} 21$, plays a key

\footnotetext{
*Correspondence: jcLu@gzhmc.edu.cn; chenkexin@tjmuch.com; zhengli@ tijmu.edu.cn

${ }^{\dagger}$ Equal contributors

${ }^{5}$ The Institute for Chemical Carcinogenesis, State Key Lab of Respiratory Disease, Guangzhou Medical University, Guangzhou 510182, China 'Department of Epidemiology and Biostatistics, , Tianjin Medical University Cancer Institute and Hospital, Tianjin 300060, P. R. China ${ }^{2}$ Department of Biochemistry and Molecular Biology, Tianjin Medical University Cancer Institute and Hospital, Tianjin 300060, P. R. China Full list of author information is available at the end of the article
}

role in sustaining cell-cycle checkpoints after DNA damage to maintain the genomic stability $[1,2]$. The defects in this pathway may result in genomic instability and carcinogenesis. Over the past few years, emerging evidence have revealed a role of p53 in regulating human maternal reproduction [3]. It is well-known that reproductive history represents lifetime exposure to hormones and is a significant risk factor for breast cancer, besides the family history of breast cancer [4-9]. However, whether the modulation of p53 activation may contribute to the genetic basis underlying the effect of reproductive history on the risk of breast cancer remains unknown.

Recently, the fragile-site associated tumor suppressor (FATS, aka C10orf90), a regulator of p53-p21 pathway, has been identified at a common fragile site (CFS) FRA10F mapped to 10q26, a genomic region susceptible to DNA damage and frequently deleted in tumor genomes [10-13]. 
Our previous reports show that the deficiency of FATS mRNA is observed in multiple cancer cell lines and clinically relevant to human cancers including breast cancer [11, $14,15]$. More recently, we reveal that the $\mathrm{NH} 2$-terminus of FATS exhibits a unique ubiquitin ligase (E3) activity to promote p53 activation in response to DNA damage [12]. The p53-p21 pathway plays a key role in sustaining cell-cycle checkpoints after DNA damage [1, 2], and the expression of the largest exon of FATS is sufficient to activate p53-p21 pathway and suppress tumorigenesis [10-12]. However, whether the non-proteolytic ubiquitination of p 53 by FATS may have some physiologic significance and whether the genetic variants of FATS may modulate the risk of breast cancer remain unknown.

In this study, we set out to test the hypothesis that FATS genetic variant may predispose to breast cancer development using a population-based study design coupled with functional validation.

\section{Methods}

\section{Study subjects}

The study population consisted of two independent cohorts: the Tianjin cohort (Discovery cohort) included 1532 patients with newly diagnosed and histologically confirmed breast cancer and 1573 age-matched ( \pm 5 years) healthy female controls, randomly extracted from a populationbased case-control study we have set up [16]. As a Replication cohort (Guangzhou cohort), 804 newly diagnosed and histopathologically confirmed breast cancer patients were consecutively recruited between March 1st, 2007 and March 1st, 2011 from four urban hospitals (i.e., the First, the Second and the Tumor Hospitals affiliated to Guangzhou Medical University, and Guangzhou Chest Hospital) and one suburban hospital, Panyu People's Hospital, with a response rate of $91 \% .835$ age-matched ( \pm 5 years) healthy controls were recruited from Guangzhou, a city in South China, with a response rate of about $85 \%$.

All participants enrolled in this study were of Chinese Han ethnicity. The Ethics Committee of Tianjin Medical University Cancer Hospital (TMUCIH) and Guangzhou Medical University (GMU) approved the study protocol, and we obtained written informed consent from all patients and controls to participate in this study.

Each subject filled out a questionnaire about demographics, menstrual and reproductive history, environmental exposures, lifestyle, and family history of cancer. Each subject donated $20 \mathrm{~mL}$ of blood that was collected into heparinized tubes and used for DNA extraction and genotyping. Paired normal tissue samples (the distance from tumor tissue: $>5 \mathrm{~cm}$ ) randomly selected from the breast cancer cases included in the study were verified by pathology specialists, and prepared for genotyping and FATS expression quantification. The tissue samples for these selected cases were obtained from the Tissue
Bank Facility of TMUCIH with approval of the Institutional Review Board (IRB).

\section{Quantitative RT-PCR}

The total RNA was extracted for cDNA synthesis, and quantitative real-time PCR was performed as described previously [15]. Briefly, Total RNA (5 $\mu \mathrm{g})$ was transcribed into complementary DNA (Invitrogen kit) and subjected to quantitative RT-PCR analysis. The primers and Taqman probes of FATS (C10orf90) (GenBank accession number: NM_001004298) were 5'-CATTCACATTCCTGGCTGG AGTTA-3', 5'-CCTCTTGCTGCTTCCAGAAAATACT-3', and 5' (FAM)-CAGGGCAGTACACACAAA-(TAMRA)3'. The primers and Taqman probes of GAPDH were 5'GAAGGTGAAGGTCGGAGTC-3', 5'-GAAGATGGTGA TGGGATTTC-3', and 5'(FAM)-CAAGCTTCCCGTTCT CAGCC-(TAMRA)-3'. Assays were carried out using the ABI 7500 TaqMan system (Applied Biosystems). Quantification of FATS gene expression in each sample was determined by measuring PCR cycle number at which the amount of FATS transcripts reached a fixed threshold $\left(C_{T}\right)$. The average $C_{T}$ value for FATS gene in each sample was obtained from three independent experiments, and normalized by that of GAPDH gene to obtain $\Delta \mathrm{C}_{\mathrm{T}} . \Delta \mathrm{C}_{\mathrm{T}}=\mathrm{C}_{\mathrm{T}}$ (FATS)- $\mathrm{C}_{\mathrm{T}}$ (GAPDH). The quantity of FATS mRNA in each sample was calculated as $2^{-\Delta \mathrm{CT}}$.

\section{Immunohistochemical analysis}

Formalin-fixed and paraffin embedded blocks of 30 breast cancer specimens and paired normal tissues available were analyzed using a rabbit polyclonal FATS (C10orf90) antibody (Abcam, ab122497). Briefly, deparaffinized sections were boiled for $15 \mathrm{~min}$ in a $1-\mathrm{mM}$ sodium citrate buffer (pH 6.0) for antigen retrieval. After quenching of endogenous peroxidases with $0.3 \%$ hydrogen peroxide in methanol, followed by two rinses with Tris- $\mathrm{HCl}$ buffer, the sections were incubated with the anti-C10orf90 antibody diluted 1:500 overnight at $4{ }^{\circ} \mathrm{C}$. Biotinylated goat anti-rabbit antibody was used as the secondary antibody and developed with liquid DAB substrate chromogen system (Dako). Hematoxylin was used for nuclear counterstaining; the sections were then mounted and coverslipped. The immunohistochemical expression of FATS was evaluated by microscope imaging. Histology analyses were evaluated in a blinded fashion by two pathologists.

\section{Single nucleotide polymorphism (SNP) identification, selection and genotyping}

With reference to the resequencing data of 45 Chinese Han individuals in the International HapMap Project SNP database (http://www.hapmap.org) and National Center for Biotechnology Information (NCBI) dbSNP database (http:// www.ncbi.nlm.nih.gov/SNP), we selected twelve SNPs with the minor allele frequency $(\mathrm{MAF})>0.01$ reported within 
the $1.0 \mathrm{~kb}$ promoter region, 5'-UTR, coding region, and 3'UTR of FATS (C10orf90) gene (Additional file 1). Genomic DNA was extracted from the whole blood using a DNA Blood Mini Kit (QIAGEN), according to the manufacturer's instructions. These SNPs were validated by DNA sequencing of PCR products in 30 randomly selected healthy subjects (Tianjin cohort). At present study, we preferentially selected one SNP (rs11245007) located in the 3rd coding exon of FATS which is responsible for the major function of FATS protein, with the highest MAF of 0.44 and the most possibly putative functional potential to genotype. Genotyping was performed by using the MGB TaqMan probe assay (Applied Biosystems Inc. [ABI], Foster City, CA). The concordance rate for genotypes was $100 \%$ in $10 \%$ of samples with duplicates.

\section{Cell culture}

The human breast cancer cell line MCF-7 was obtained from the American Type Culture Collection (ATCC) in 2008. The cell line has been last tested and authenticated in 2013 by genetic profiling using the well-known short tandem repeat (STR) loci [17]. The cell line was maintained in culture as an adherent monolayer in DMEM (Invitrogen) medium supplemented with 10 \% FBS. Cells were incubated at $37{ }^{\circ} \mathrm{C}$ in a humidified atmosphere with $5 \% \mathrm{CO} 2$.

\section{Vectors and site-specific mutagenesis}

The expression vector CMV-C10orf90, i.e. FATS-262D, was purchased from Origene. Flag-FATS-262D plasmid was constructed by in-frame inserting full-length FATS cDNA into p3xFlag-myc-CMV-26 vector (Sigma). FlagFATS-262 $\mathrm{N}$ was generated by site-directed mutagenesis, according to manufacturer's instructions (Stratagene). The primer sequences are 5'-GTCTCAGCAGTGTCCCGATG CCATTTACTATTTGG-3' and 5'-CCAAAT AGTAAATG GCATCGGGACACTGCTGAGAC-3'. FATS cDNA was in-frame inserted into pGEX-6p-1 vector (GE Healthcare Life Sciences) to generate GST-FATS-262D or GSTFATS-262 N, respectively.

GST-FATS-262 N/ GST-FATS-262D was transformed into Escherichia coli BL21. The bacteria were grown at $30{ }^{\circ} \mathrm{C}$ in LB medium, and GST-fusion protein synthesis was induced with 0.5 to $1.0 \mathrm{mmol} / \mathrm{L}$ of isopropyl-l-thio$\beta$-D-galactopyranoside. Cells were harvested after 3 to 4 hours. The cell pellet was resuspended in cold sodiumTris-EDTA lysis buffer $[10 \mathrm{mmol} / \mathrm{L}$ Tris $(\mathrm{pH} \quad 8.0)$, $1 \mathrm{mmol} / \mathrm{L}$ EDTA, and $150 \mathrm{mmol} / \mathrm{L} \mathrm{NaCl}$ supplemented with lysozyme (1 mg/mL; Sigma) and incubated on ice for 15 minutes. Just before sonication, $1 \mathrm{mmol} / \mathrm{L}$ DTT, $10 \mathrm{mmol} / \mathrm{L} \mathrm{MgCl} 2,1 \mathrm{mmol} / \mathrm{L}$ phenylmethylsulfonylfluoride (PMSF), and $1 \%$ Sarkosyl (Sigma) were added to the cell lysate and mixed thoroughly. The cell lysate then was sonicated at maximum intensity for 20 seconds.
Triton X-100 (2\%) was added, and the cell lysate was mixed gently for 30 minutes to help the fusion protein dissolve. After centrifugation, the GST-fusion protein was purified by Glutathione Sepharose 4B (Amersham Biosciences) and eluted with buffer $[50 \mathrm{mmol} / \mathrm{L}$ Tris ( $\mathrm{pH}$ 8.0)] containing $10 \mathrm{mmol} / \mathrm{L}$ glutathione (reduced form; Sigma).

MCF-7 cells were transfected with plasmid DNA using a Nucleofector kit (Amaxa) or Lipofectamine 2000 (Invitrogen), according to manufacturer's instructions.

\section{Cell fractionation}

Cells were washed with cold PBS twice and collected in lysis buffer $[20 \mathrm{mmol} / \mathrm{L}$ HEPES ( $\mathrm{pH} 7.5), 10 \mathrm{mmol} / \mathrm{L}$ $\mathrm{KCl}, 2 \mathrm{mmol} / \mathrm{L} \mathrm{MgCl} 2,0.5 \% \mathrm{NP} 40,100 \mathrm{mmol} / \mathrm{L} \mathrm{NaF}$, $1 \mathrm{mmol} / \mathrm{L} \mathrm{Na} 3 \mathrm{VO} 4,1 \mathrm{mmol} / \mathrm{L}$ PMSF, and $1 \%$ aprotinin]. Let cells sit in lysis buffer on ice for 30 minutes to ensure complete lysis. Spin cells at $12000 \mathrm{~g}$ for 10 minutes at $2-4{ }^{\circ} \mathrm{C}$. The supernatant was saved as whole cell lysates.

\section{Immunoblotting assay}

The cell lysates were separated by SDS-PAGE and transferred to PROTRAN nitrocellulose membranes (Schleicher \& Schuell, Dassel, Germany). For immunoblot experiments, 20 to $50 \mu \mathrm{g}$ of lysates in SDS loading buffer were separated by SDS-PAGE. Western blot analysis was exposured to $\mathrm{X}$-ray film for autoradiography.

\section{Dual luciferase reporter assay}

Cells $\left(5 \times 10^{4}\right)$ were transfected with $250 \mathrm{ng}$ of firefly luciferase reporter (pGL3-FATS-luc), $20 \mathrm{ng}$ of the transfection control Renilla vector (pRL-TK) and $100 \mathrm{ng}$ of p53-expressing vector in combination with $500 \mathrm{ng}$ of FATS-expressing vector. At $24 \mathrm{~h}$ after transfection, cells were lysed in 1x passive lysis buffer (Promega), and reporter activity was measured using the Dual-Luciferase Reporter Assay System (Promega). Each assay was tested in triplicate in three independent experiments.

\section{Ubiquitination assay}

The ubiquitination assay was performed as described previously [12]. In brief, purified GST-FATS-262 N/ GST-FATS-262D $(1 \mu \mathrm{g})$, E1 (40 ng, Calbiochem or Sigma), and ubiquitin ( $2 \mu \mathrm{g}$, Boston Biochem) was incubated with in vitro translated p53 protein in $30 \mu \mathrm{l}$ reaction buffer $(50 \mathrm{mM}$ Tris- $\mathrm{HCl}[\mathrm{pH}$ 7.4], $2 \mathrm{mM}$ ATP, $5 \mathrm{mM} \mathrm{MgCl}_{2}$, $2 \mathrm{mM}$ DTT, $30 \mathrm{mM}$ creatine phosphate, and $0.05 \mathrm{mg} / \mathrm{ml}$ creatine phosphokinase) at $30{ }^{\circ} \mathrm{C}$ for $90 \mathrm{~min}$. The reactions were stopped by adding $2 \times$ SDS loading buffer and heating at $95{ }^{\circ} \mathrm{C}$ for $5 \mathrm{~min}$. Ubiquitinated p53 proteins were detected by immunoblotting using a p53-specifc antibody. In the absence of p53, the assembly of poly-ubiquitin was examined by Western blot using an ubiquitin antibody. 


\section{Statistical analysis}

The Kruskal-Wallis H Test was used to analyze the differences of mRNA expression between breast tumor samples and normal breast tissues. A $x^{2}$ test was used to examine the differences in demographic variables and genotype distribution of FATS polymorphisms between patients and controls. A Hardy-Weinberg equilibrium test was performed for the genotype distribution in the controls to evaluate possible selection bias and genotyping errors. The multivariate logistic regression method was used to assess the association between breast cancer risk and FATS gene SNP. Odds ratios (ORs) and $95 \%$ confidence intervals (CIs) were calculated with adjustment for known risk factors of breast cancer, such as age, BMI, age at menarche, birth number, duration of breast-feeding, menopause status, oral contraception, smoking status (ever/never), exercise, benign breast disease, and family history of cancer (first/second degree). For cases only, we also performed stratified case-series analysis of the genotype data by clinical phenotypes. All statistical tests were two-sided and a $P$ value $<0.05$ was considered statistically significant using SAS v9.0 software (Cary, NC, USA).

Heterogeneity of the association between FATS SNP and breast cancer risk from the Discovery and Replication cohorts was estimated by the $I^{2}$, which was ranked as "no-" (0\% $\left.\leq I^{2}<25 \%\right)$, "moderate-" $\left(25 \% \leq I^{2}<50 \%\right)$, "large-" (50\% $\left.\leq I^{2}<75 \%\right)$ and "extreme-" (75 \% $\leq I^{2} \leq$ $100 \%)$ heterogeneity between these two cohorts (Marcos et al., 2009). A random-effects model (DerSimonian and Laird method) or fixed-effects model (Mantel-Hansel method) was used to calculate the pooled OR in the presence $(P \leq 0.10)$ or absence $(P>0.10)$ of heterogeneity, respectively [18, 19]. Analyses were conducted using Stata 11.0.

\section{Results}

\section{Functional characterization of a genetic variant}

We first validated the clinical relevance of FATS expression to breast cancer. Consistent to the sample set from our previous report [14], we confirmed that the expression level of FATS mRNA was silent or down-regulated in $100 \%$ matched breast tumor tissues $(\mathrm{n}=38)$, compared with that in normal tissues (Fig. 1a). Consistently, the expression of FATS protein was downregulated in $73.3 \%$ tested breast cancer samples in comparison to that in normal breast tissues (Fig. 1b). We further measured the mRNA levels of FATS in breast tumor tissues $(\mathrm{n}=156)$ and found that the average level of FATS mRNA even in pathologic stage-I breast tumors was 10-fold lower than that in normal control $(P<0.01$, Fig. $1 c)$ and were inversely correlated with pathological stages (extensively downregulated or nearly silent in stage III), suggesting that FATS deficiency occurred at the early stage of tumorigenesis. These results, in combination with our previous functional studies on FATS [10-12], raised the possibility that the SNP in FATS may modulate the risk of breast cancer.

To test the hypothesis that SNP in FATS gene may contribute to the susceptibility of breast cancer, we firstly evaluated those potentially functional SNPs in FATS (Additional file 1). Human FATS (C10orf90) gene contains 9 exons whose coding protein consists of 699 amino acid residues (Fig. 2a). The power to detect genetic effects is dependent on MAF. There are only 4 SNPs in FATS exons with MAF $>0.01$ and missense function (Fig. 2b). Notably, a SNP with MAF $>0.25$ is located at the largest coding exon of FATS, which is responsible for the major function of FATS protein [10-12]. This SNP, rs11245007 $(905 \mathrm{C}>\mathrm{T})$, with a MAF value of 0.4440 and causing $262 \mathrm{D} / \mathrm{N}$ substitution, was confirmed by DNA sequencing in Chinese Han population (Fig. 2c). Interestingly, $262 \mathrm{D} / \mathrm{N}$ is only one amino-acid away from the conserved catalytic Cys residue of FATS protein as an E2-independent E3 (Fig. 2D), raising the probability that FATS-262 N may differ from FATS-262D in its E3 activity. Indeed, the results of ubiquitination assay indicated that the E3 activity of FATS-262 $\mathrm{N}$ to assemble polyubiquitin chains was significantly stronger than that of FATS-262D in an E2-independent manner (Fig. 3a).

Because FATS-catalyzed non-proteolytic polyubiquitination of p53 is required for robust activation of p53 in response to DNA damage [12], we further investigated whether FATS-262D or FATS-262 N may cause different modification status of p53 polyubiquitination. As shown in Fig. 3b, the polyubiquitination of p53 by FATS-262 N was more pronounced than that of FATS-262D. Consistently, FATS-262 N exhibited a stronger effect on stimulating the transcriptional activity of p53 (Fig. 3c) and facilitating the stabilization of p53 protein in response to DNA damage (Fig. 3d). Meanwhile, the acetylation and phosphorylation of p53, which are detectable after DNA damage and coupled with p53 activation, was more remarkable in the presence of FATS-262 $\mathrm{N}$ than in the presence of FATS-262D (Fig. 3d). Therefore, rs11245007 $(905 \mathrm{C}>\mathrm{T}, 262 \mathrm{D} / \mathrm{N})$ was a functional SNP with impact on p53 activation.

\section{Population-based analysis of the SNP rs11245007}

The functional identification of rs11245007 (905C > T, $262 \mathrm{D} / \mathrm{N}$ ) in FATS prompted us to test its genetic effect on breast cancer risk. We performed a case-control study (Discovery Cohort) that included 1532 breast cancer cases and 1573 healthy controls. The study subjects in this cohort were all recruited from Tianjin, a metropolis in North China (Additional file 2). As expected, patients in the discovery set reported a greater number of known risk factors for breast cancer than did the controls. For 


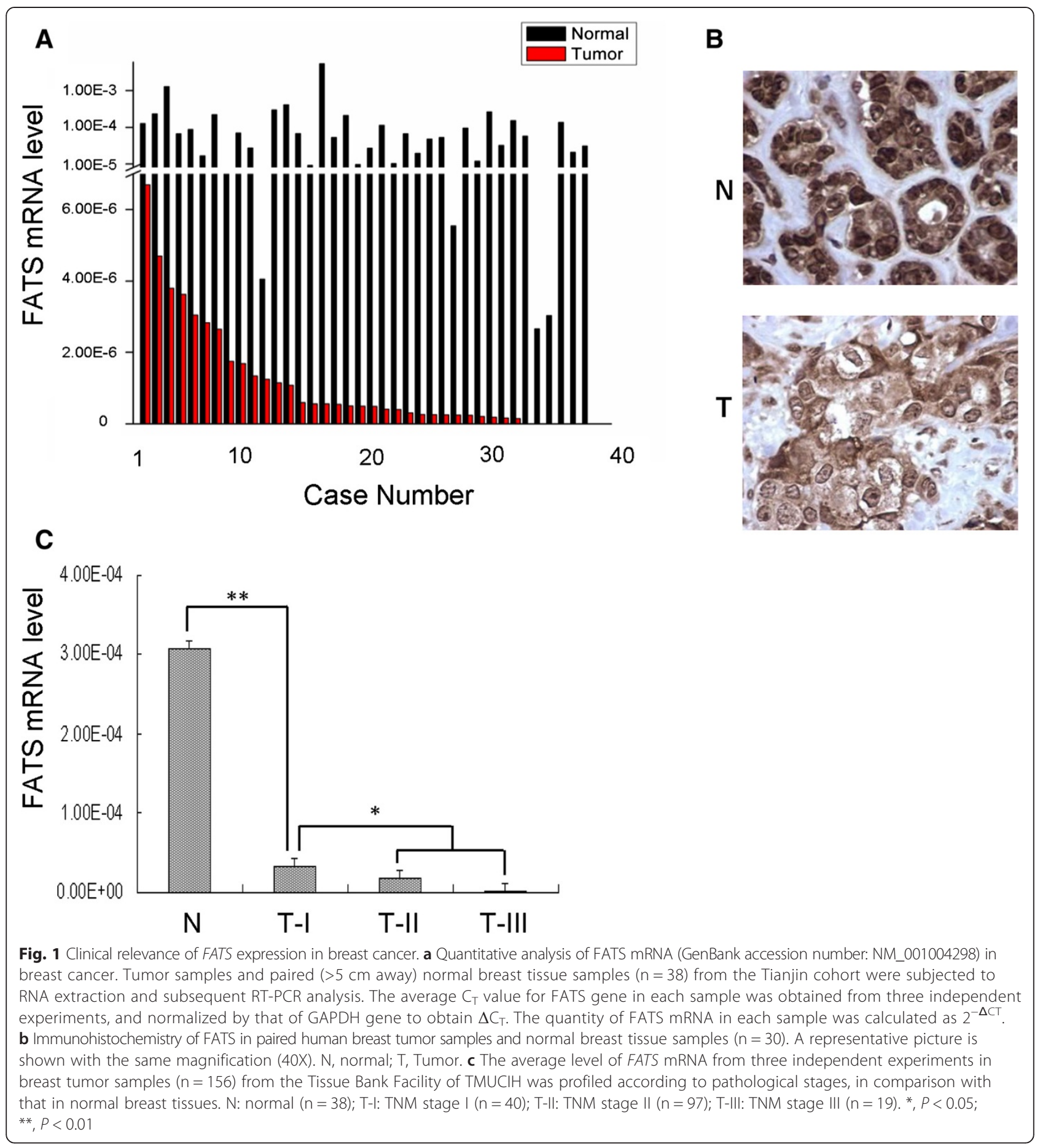

example, significantly larger proportions of patients than the controls had fewer birth numbers, less breast-feeding time and physical activity, were nulliparous and younger at menarche, and had history of benign breast disease, and family history of cancer. Genotype frequencies among the controls did not show significant departures from HardyWeinberg equilibrium $(P=0.407)$. We did not observe a difference in the genotypic frequencies of rs11245007 between patients and controls $(P=0.907)$, and the results of a case-only analysis indicated that the rs 11245007 genotypes were not significantly associated with mean age at diagnosis, lymph node metastasis, and expression status of estrogen receptor (ER) or progesterone receptor (PR) (Additional file 3). In Discovery cohort, the rs11245007 TT or TT + CT genotypes did not show a significant protective effect on overall risk of breast cancer, 


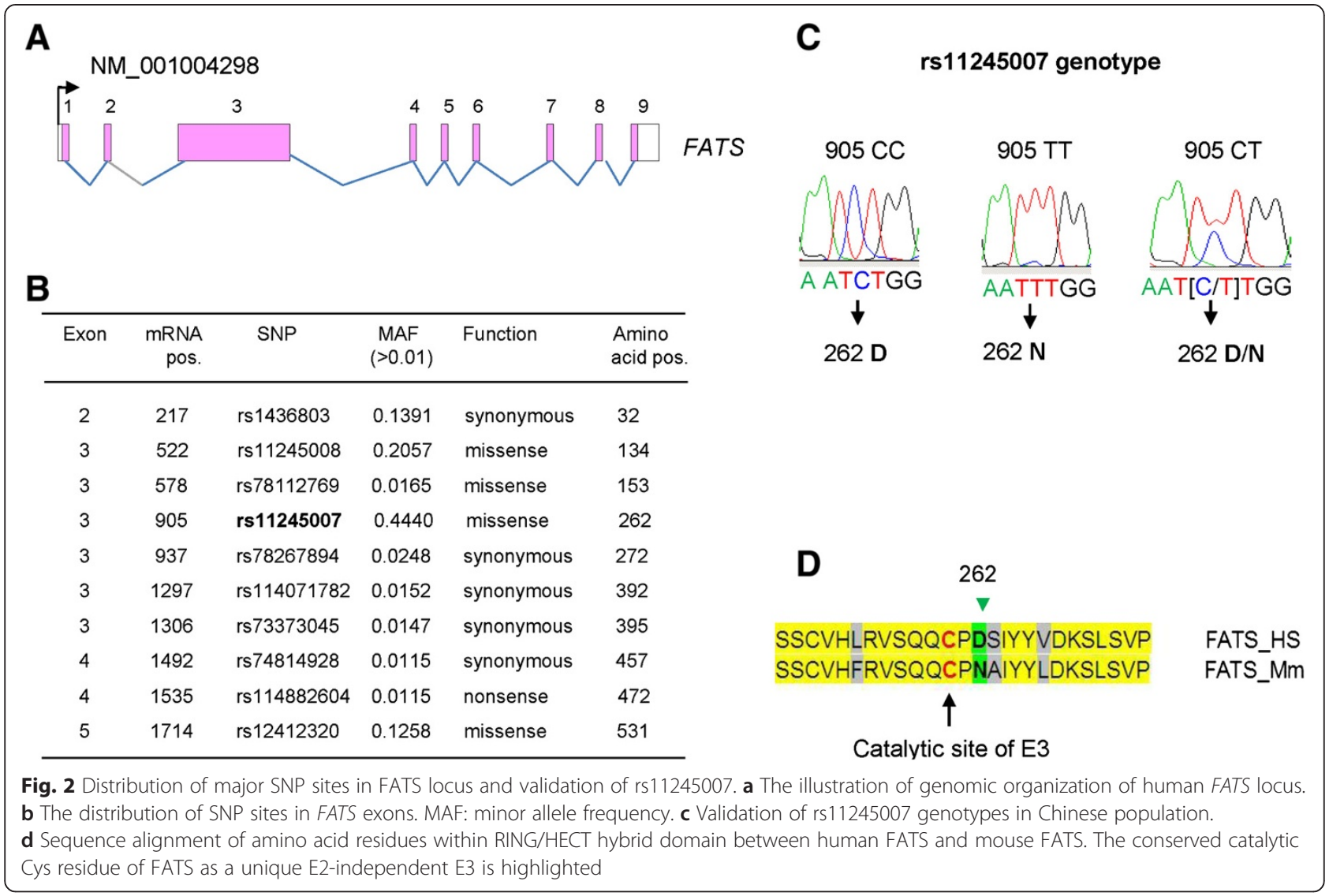

although the CT genotype was associated with a decreased overall risk of breast cancer compared with the CC genotype (OR, 0.796; $95 \%$ CI, 0.638-0.992; $P=0.042$ ) (Table 1).

However, when the results were further stratified by parity (birth number), we found that both the rs11245007 CT and TT genotypes were associated with a decreased risk of breast cancer in subjects who had given birth more than three times, compared with the CC genotype. When combined together, the protective effect of the $\mathrm{T}$ allele (CT + TT genotype) was more pronounced in subjects with parity $>=3$ (OR, 0.499; $95 \% \mathrm{CI}, 0.307-0.813 ; P=$ 0.0053 ) (Table 1). For those with the family history of cancer (positive family history of cancer in first- and seconddegree relatives), the CT genotype was also associated with a decreased breast cancer risk (OR, 0.530; 95 \% CI, 0.2990.937; $P=0.0289$ ), compared with the CC genotype. Paradoxically, the TT genotype was not significantly associated with a decreased breast cancer risk in women with family history (OR, 0.592; $95 \% \mathrm{CI}, 0.304-1.151 ; P=0.1223$ ) (Table 1).

To verify our findings that rs11245007 modulated breast cancer risk in women with parity $\geq 3$ and clarify the effect of rs11245007 on breast cancer risk in women with family history, we further perform an independent case-control analysis (Replication cohort). The subjects in this cohort were recruited from Guangzhou, a major city in South China where is 1493 miles away from Tianjin, excluding the probability of repetitive recruitment (Additional file 4). Some differences from the discovery set in the distributions of the aforementioned baseline variables between cases and controls were observed in the validation set, even that the cases had a higher percentage of known breast cancer factors, such as family history of cancer and menstruation. These possibly resulted from the relatively smaller sample number of this cohort (just over half of the discovery cohort). The controls in this cohort showed a higher proportion of early menarche (10.8 vs. $4.21 \%)$ and infertility (6.70 \% vs. $1.34 \%)$ than those in the discovery cohort. indicating some selection bias in controls of the replication set as well as the geographic variance in economy and culture. Consistently, there were no significant associations between the rs11245007 genotypes and patients clinical features including lymph node metastasis and expression status of ER or PR in Replication cohort (Additional file 5).

In concert with results of the case-control study in Discovery cohort (Table 1), the rs11245007 genotypes were not significantly associated with an overall protective effect on breast cancer risk in Replication cohort. Remarkably, the protective effect of the $905 \mathrm{~T}$ allele $(\mathrm{CT}+\mathrm{TT})$ on 


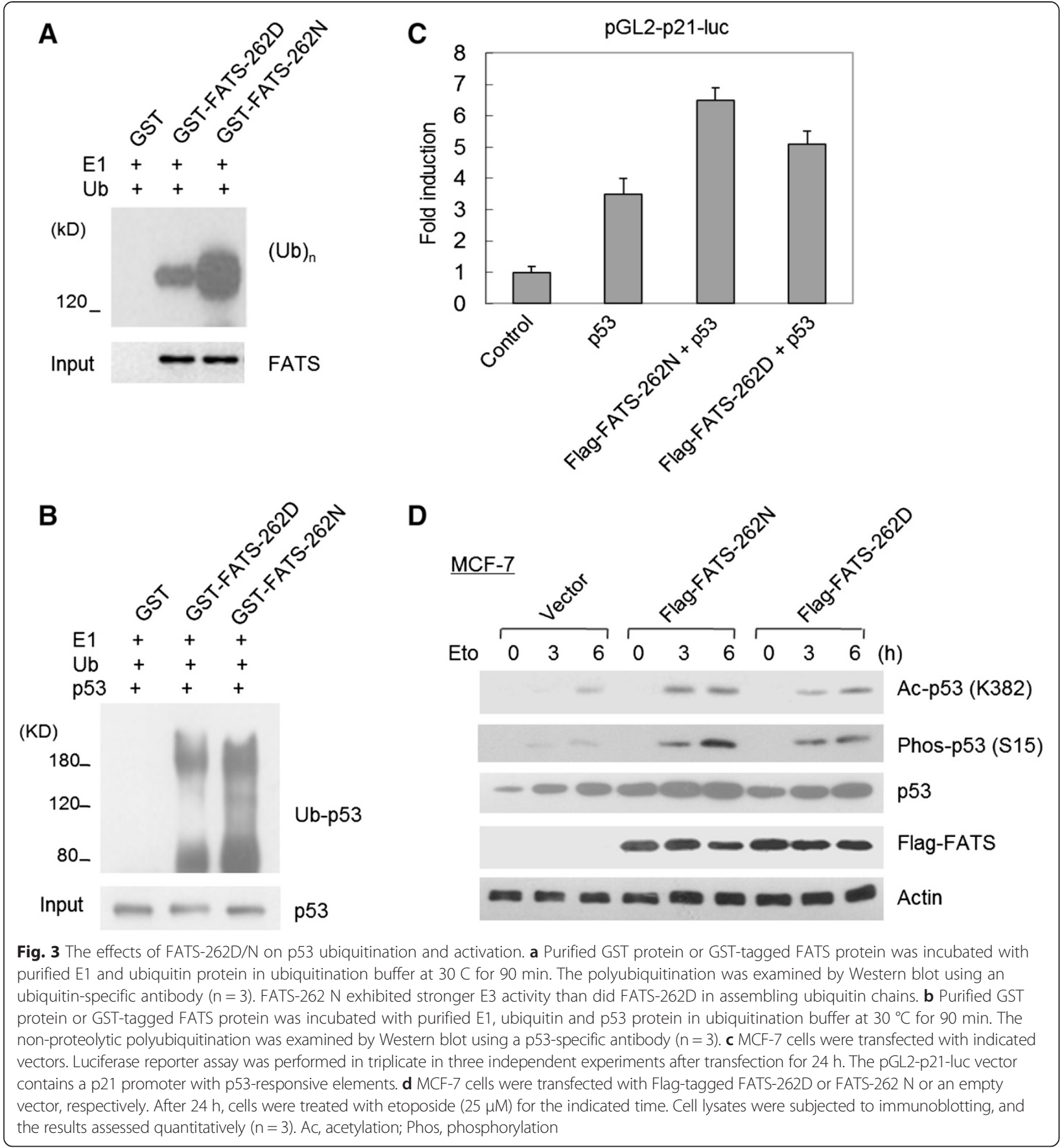

breast cancer risk was still statistically significant in subjects with parity $\geq 3$ (adjusted OR $=0.558$; $95 \% \mathrm{CI}$ : $0.363-0.857 ; P=0.0077)$, and such protective effect was significant for $\mathrm{CT}$ (adjusted $\mathrm{OR}=0.547,95 \% \mathrm{CI}$ : $0.343-0.872 ; P=0.0113$ ) and TT (adjusted $\mathrm{OR}=0.579$, 95 \% CI: $0.336-0.997 ; P=0.0492$ ) genotypes in triparous women (Table 2). The association between rs11245007 genotypes and family history of cancer in Replication cohort was not statistically significant (Table 2).
Considering the sampling error and random error due to geographic difference between these two cohorts, meta-analyses were used to evaluate the potential heterogeneity between these two cohorts and to calculate the pooled association between SNP and breast cancer risk with ORs from these two cohorts (shown in Additional file 6). As expected, there were no heterogeneities observed in the two cohorts, for both the overall and stratified analysis on the association between FATS SNP genotypes and 
Table 1 Overall and stratified analyses on the association of rs11245007 genotypes with risk of breast cancer in Discovery cohort by multivariate logistic regression model

\begin{tabular}{|c|c|c|c|c|}
\hline \multirow[t]{2}{*}{ Genotype } & \multicolumn{4}{|c|}{ rs11245007 (C/T) } \\
\hline & $\mathrm{CC}$ & $\mathrm{CT}$ & $\pi$ & $C T+T T$ \\
\hline \multicolumn{5}{|l|}{ All subjects } \\
\hline Cases $(n=1532)$ & $432(28.20)$ & $738(48.17)$ & $362(23.63)$ & $1100(71.80)$ \\
\hline Controls $(n=1573)$ & $443(28.16)$ & $768(48.82)$ & $362(23.01)$ & $1130(71.84)$ \\
\hline Crude OR (95 \% Cl) & 1.00 & $0.985(0.834,1.164)$ & $1.025(0.842,1.249)$ & $0.998(0.854,1.167)$ \\
\hline$P$ & & 0.863 & 0.802 & 0.982 \\
\hline Adjusted OR $(95 \% \mathrm{Cl})^{\mathrm{a}}$ & 1.00 & $0.796(0.638,0.992)$ & $0.952(0.735,1.235)$ & $0.844(0.687,1.037)$ \\
\hline P & & 0.0420 & 0.7124 & 0.1069 \\
\hline \multicolumn{5}{|l|}{ Parity $<3^{b}$} \\
\hline Cases $(n=1293)$ & $347(26.84)$ & $636(49.19)$ & $310(23.98)$ & $946(73.16)$ \\
\hline Controls ( $n=1122)$ & $314(27.99)$ & $564(50.27)$ & $244(21.75)$ & $808(72.01)$ \\
\hline Adjusted OR $(95 \% \mathrm{Cl})^{\mathrm{a}}$ & 1.00 & $0.962(0.751,1.232)$ & $1.324(0.987,1775)$ & $1.066(0.846,1.345)$ \\
\hline$P$ & & 0.7605 & 0.0607 & 0.5865 \\
\hline \multicolumn{5}{|l|}{ Parity $\geq 3^{b}$} \\
\hline Cases $(n=188)$ & $67(35.64)$ & $81(43.09)$ & $40(21.28)$ & $121(64.36)$ \\
\hline Controls $(n=426)$ & $119(27.93)$ & $194(45.54)$ & $113(26.53)$ & $307(72.07)$ \\
\hline Adjusted OR $(95 \% \mathrm{Cl})^{\mathrm{a}}$ & 1.00 & $0.517(0.304,0.879)$ & $0.469(0.251,0.876)$ & $0.499(0.307,0.813)$ \\
\hline$P$ & & 0.0148 & 0.0175 & 0.0053 \\
\hline \multicolumn{5}{|l|}{ Family history of cancer ${ }^{c}$} \\
\hline Cases $(n=474)$ & $126(26.58)$ & $238(50.21)$ & $110(23.21)$ & $348(73.42)$ \\
\hline Controls $(n=169)$ & $31(18.34)$ & $95(56.21)$ & $43(25.44)$ & $138(81.66)$ \\
\hline Adjusted OR $(95 \% \mathrm{Cl})^{\mathrm{a}}$ & 1.00 & $0.530(0.299,0.937)$ & $0.592(0.304,1.151)$ & $0.548(0.317,0.946)$ \\
\hline$P$ & & 0.0289 & 0.1223 & 0.0308 \\
\hline \multicolumn{5}{|c|}{ No family history of cancer ${ }^{c}$} \\
\hline Cases $(n=1057)$ & $306(28.95)$ & $500(47.30)$ & $251(23.75)$ & $751(71.05)$ \\
\hline Controls $(n=1401)$ & $411(29.34)$ & $672(47.97)$ & $318(22.70)$ & $990(70.66)$ \\
\hline Adjusted OR $(95 \% \mathrm{Cl})^{\mathrm{a}}$ & 1.00 & $0.919(0.723,1.166)$ & $1.130(0.854,1.494)$ & $0.985(0.788,1.230)$ \\
\hline$P$ & & 0.4860 & 0.3933 & 0.8908 \\
\hline
\end{tabular}

Abbreviations: OR Odds ratios; $\mathrm{Cl}$ confidence interval

${ }^{a} \mathrm{OR}$ is adjusted for age, BMI, menarche age, parity, time of breast feeding, menopause, oral contraception, smoking status (ever/never), exercise, benign breast disease, and family history of cancer (first- and second-degree relatives)

${ }^{b}$ due to missing values, $\mathrm{n}$ (total cases with parity data) $<1532$, n (total controls with parity data) $<1573$

'due to missing values, $\mathrm{n}$ (total cases with family history of cancer data) $<1532, \mathrm{n}$ (total controls with family history of cancer data) $<1573$

breast cancer risk (all $P>0.10$ ). Genotypes with T-allele (CT + TT genotype) was associated with a decreased overall risk of breast cancer compared with the CC genotype $(\mathrm{OR}=0.84 ; 95 \% \mathrm{CI}: 0.71-0.97)$. Further, this protective effect of the $\mathrm{T}$ allele was more pronounced in subjects with parity $>=3(\mathrm{OR}=0.53 ; 95 \% \mathrm{CI}: 0.35-0.71)$ and with family history of cancer (OR $=0.51 ; 95 \%$ CI: $0.25-0.77)$.

\section{Discussion}

In this study, we first revealed that a SNP rs11245007, which is located in the largest exon of FATS, is functional in facilitating p53 activation. Two independent case-control studies validated that rs 11245007 is an important genetic variant affecting the susceptibility to parity-dependent breast cancer.

Breast tissues undergo extensive physiologic changes during full-term pregnancy, which may vulnerable to breast carcinogenesis. Two of the earliest known and most reproducible features of breast cancer epidemiology are the decreased risk associated with parity and early age at first full-term pregnancy. The long-term protective effect of a term birth on breast cancer risk is preceded by a short-term adverse effect, possibly reflecting a promoting effect of pregnancy hormones. The 
Table 2 Overall and stratified analyses on the association of rs11245007 genotypes with risk of breast cancer in Replication cohort by multivariate logistic regression model

\begin{tabular}{|c|c|c|c|c|}
\hline \multirow[t]{2}{*}{ Genotype } & \multicolumn{4}{|c|}{ rs11245007 (C/T) } \\
\hline & $\mathrm{CC}$ & $C T$ & $\pi$ & $C T+\pi$ \\
\hline \multicolumn{5}{|l|}{ All subjects } \\
\hline Cases $(n=804)$ & $243(30.22)$ & $364(45.27)$ & $197(24.50)$ & $561(69.78)$ \\
\hline Controls $(n=835)$ & $223(26.71)$ & $392(46.95)$ & $220(26.35)$ & $612(73.29)$ \\
\hline Crude OR (95 \% Cl) & 1.00 & $0.852(0.676,1.074)$ & $0.822(0.631,1.071)$ & $0.841(0.679,1.043)$ \\
\hline$P$ & & 0.1748 & 0.1460 & 0.1148 \\
\hline Adjusted OR $(95 \%$ Cl) & 1.00 & $0.863(0.668,1.115)$ & $0.789(0.587,1.060)$ & $0.836(0.659,1.061)$ \\
\hline P & & 0.2601 & 0.1158 & 0.1405 \\
\hline \multicolumn{5}{|l|}{ Parity $<3^{b}$} \\
\hline Cases $(n=468)$ & $129(27.56)$ & $218(46.58)$ & $121(25.85)$ & 339 (72.44) \\
\hline Controls $(n=505)$ & $137(27.13)$ & $230(45.54)$ & $138(27.33)$ & $368(72.87)$ \\
\hline Adjusted OR $(95 \% \mathrm{Cl})^{\mathrm{a}}$ & 1.00 & $1.059(0.774,1.449)$ & $0.922(0.646,1.318)$ & $1.008(0.753,1.349)$ \\
\hline$P$ & & 0.7209 & 0.6571 & 0.9590 \\
\hline \multicolumn{5}{|l|}{ Parity $\geq 3^{b}$} \\
\hline Cases $(n=234)$ & $92(39.32)$ & $93(39.74)$ & $49(20.94)$ & $142(60.68)$ \\
\hline Controls $(n=224)$ & $62(27.68)$ & $107(47.77)$ & $55(24.55)$ & $162(72.32)$ \\
\hline Adjusted OR (95\% Cl) ${ }^{\mathrm{a}}$ & 1.00 & $0.547(0.343,0.872)$ & $0.579(0.336,0.997)$ & $0.558(0.363,0.857)$ \\
\hline$P$ & & 0.0113 & 0.0492 & 0.0077 \\
\hline \multicolumn{5}{|l|}{ Family history of cancer } \\
\hline Cases $(n=81)$ & $23(28.40)$ & $33(40.74)$ & $25(30.86)$ & $58(71.60)$ \\
\hline Controls $(n=56)$ & $11(19.64)$ & $27(48.21)$ & $18(32.14)$ & $45(80.36)$ \\
\hline Adjusted OR (95\% Cl) $)^{\mathrm{a}}$ & 1.00 & $0.477(0.179,1.270)$ & $0.390(0.134,1.135)$ & $0.440(0.178,1.089)$ \\
\hline$P$ & & 0.1384 & 0.0839 & 0.0759 \\
\hline \multicolumn{5}{|c|}{ No family history of cancer } \\
\hline Cases $(n=723)$ & $220(30.43)$ & $331(45.78)$ & $172(23.79)$ & $503(69.57)$ \\
\hline Controls $(n=779)$ & $212(27.21)$ & $365(46.85)$ & $202(25.93)$ & $567(72.79)$ \\
\hline Adjusted OR (95\% Cl) ${ }^{\mathrm{a}}$ & 1.00 & $0.903(0.691,1.181)$ & $0.836(0.612,1.141)$ & $0.879(0.685,1.128)$ \\
\hline$P$ & & 0.4569 & 0.2585 & 0.3115 \\
\hline
\end{tabular}

Abbreviations: OR Odds ratios, $\mathrm{Cl}$ confidence interval

${ }^{a} \mathrm{OR}$ is adjusted for age, BMI, menarche age, parity, menopause, smoking status (ever/never), and family history of cancer (first- and second-degree relatives)

${ }^{b}$ due to missing values, $\mathrm{n}$ (total cases with parity data) $<804, \mathrm{n}$ (total controls with parity data) $<835$

short-term adverse effect of parity on breast cancer risk is much more evident in women with parity $\geq 3$ and without family history of cancer [7]. Although differentiation of breast cells after the first full-term birth makes them less susceptible to hormonal stimuli, it may not prevent a promoting/progressive effect of pregnancy hormones on breast cells that may be in the very early stages of a carcinogenic process [20]. The high risk after higher order births may be result from a "carry-over" effect of a previous birth. Despite the extensive and productive research on genetic variants and association with overall breast cancer risk, the regulatory pathway underlying parity-associated breast carcinogenesis and the genetic variants conferring susceptibility to parity-dependent risk of breast cancer remain poorly understood.
Recent data support the idea that the crosstalk between FATS and p53 may be of physiological importance for reproduction during evolution. The expression of FATS mRNA and protein is highest in testis and its protein level is second highest in ovary of mouse [10, 11]. Interestingly, our previous data also found the relevance of deficient FATS expression to the onset of breast cancer [14]. Likewise, p53 is a guardian of maternal reproduction. The sufficient expression of p53 is important not only for the implantation of fertilized eggs and prevention of preterm birth in mice, but also for the resistance to pregnancy-related mammary carcinogensis in mice [3, 21]. These facts suggest that the positive feedback loop between FATS and p53 may be indispensable for tighter surveillance of genomic stability during reproduction-related 
physiological processes. The relevance of deficient FATS expression to the onset of breast cancer and the role of p53 as a guardian of maternal reproduction support our findings that the promotion of p53 activation by FATS is important to inhibit pregnancy-related mammary carcinogenesis. Thus, genetic polymorphisms, especially SNPs in FATS, those potentially functional to the activation of $\mathrm{p} 53$ by FATS may affect the susceptibility to breast cancer.

Interestingly, FATS rs11245007 (262D/N) variant, regulating p53 function, did not affect the overall risk of breast cancer in our study population. Similarly, the genetic variants in Tp53 do not show effect on the risk of breast cancer [22], suggesting that any effect of genetic variants in Tp53 or FATS on breast cancer would be very small or possibly confined to subgroups. Indeed, although $B R C A 1$, $B R C A 2$ and TP53 mutations confer susceptibility to breast cancer, common variants in these genes have not been shown to confer measurably increased risks of breast cancer [23]. Unexpectedly, we found that the effect of FATS rs11245007 variant on breast cancer risk was confined to women with parity $\geq 3$. Both the rs $11245007 \mathrm{CT}$ and TT genotypes were associated with a decreased risk of breast cancer in subjects who had given birth more than three times as compared with the CC genotype, in both the Discovery and Replication cohorts. The stronger activation of p53 mediated by FATS $262 \mathrm{~N}$ would be physiologically important to suppress carcinogenesis of breast tissues undergoing repetitive and extensive changes during pregnancy for triparous women, and even a small protective effect of FATS-p53 signaling cascade may contribute significantly to decrease the risk of breast cancer as parity increases. The protective effect of FATS-p53 signaling cascade on breast cancer risk may be confined to the subgroup of triparous women. Although rs11245007 T allele $(\mathrm{CT}+\mathrm{TT})$ in FATS conferred a reduced risk of breast cancer in individuals with a family history of cancer in Tianjin Discovery cohort, such effect was not replicated in the Replication set of Guangzhou cohort, possibly due to the relatively smaller number of the Replication cohort, particularly for the stratification analysis.

\section{Conclusions}

In summary, we have identified for the first time a genetic variant in the FATS gene $(905 \mathrm{C}>\mathrm{T}, 262 \mathrm{D} / \mathrm{N})$ that is associated with susceptibility to breast cancer in a paritydependent manner. Functional analysis demonstrated that FATS-262 N significantly increased the p53 activity in breast cells, resulting from more pronounced polyubiquitination of $\mathrm{p} 53$ by FATS-262 N. These findings provide the emerging physiologic evidence in support of the role of FATS as an E2-independent E3 toward p53, in addition to pinpointing a genetic marker with potential value in predicting breast cancer risk in women with parity $\geq 3$. Once this genetic variant is validated by larger studies in different ethnicities, FATS genotyping for $905 \mathrm{C}>\mathrm{T}$ may have application in breast cancer prevention.

\section{Additional files}

Additional file 1: Selection for candidate FATS SNPs with the minor allele frequency (MAF) $>0.01$ reported within the $1.0 \mathrm{~kb}$ promoter region, 5'-UTR, coding region, and 3'-UTR of FATS gene. (DOCX $17 \mathrm{~kb}$ ) Additional file 2: Baseline characteristics of breast cancer cases and cancer-free controls in Discovery cohort. (DOCX $18 \mathrm{~kb}$ )

Additional file 3: Frequency distributions of FATS rs11245007 genotypes according to clinical characteristics of cases in Discovery cohort. (DOCX $18 \mathrm{~kb}$ )

Additional file 4: Baseline characteristics of breast cancer cases and cancer-free controls in Replication cohort. (DOCX $20 \mathrm{~kb}$ )

Additional file 5: Frequency distributions of FATS rs 11245007 genotypes according to clinical characteristics of cases in Replication cohort. (DOCX $18 \mathrm{~kb}$ )

Additional file 6: Forest plots describing the association between the FATS SNP (A: Genotype CT vs. CC; B: Genotype TT vs. CC; C: Genotype $C T+T T$ vs. $C C$ ) and risk of breast cancer from the Discovery and Replication cohorts. Heterogeneity from the two cohorts was estimated by the $R$, and a fixed-effects model (Mantel-Hansel method) was used to calculate the pooled $O R$ in the absence (all $P>0.10$ ) of heterogeneity. (DOCX 229 kb)

\section{Abbreviations}

FATS: Fragile-site associated tumor suppressor; CFS: Common fragile site; SNP: Single nucleotide polymorphism; MAF: Minor allele frequency; STR: Short tandem repeat; ORs: Odds ratios; Cis: Confidence intervals; ER: Estrogen receptor; PR: Progesterone receptor.

\section{Competing interests}

The authors declare that they have no competing interests.

\section{Authors' contributions}

FFS, JZ and LQ performed the experiments and statistical analysis, participated in the design of the study, and drafted the first version of the manuscript. YWZ and PX participated in the acquisition of data, helped in the interpretation of the results and to draft the manuscript. JCL, KXC and $\mathrm{ZL}$ conceived the study and supervised its design and coordination, and critically revised the manuscript for important intellectual content. All authors critically reviewed the manuscript and approved the final manuscript.

\section{Acknowledgments}

The authors thank all the patients for their willingness to participate in this study. This work was supported by grants from Ministry of Science and Technology of China 973-Program concept award [2009CB526407 to Z.L.] and 863-Program [2012AA02A207 to K.C.]; National Natural Science Foundation of China [81272283 to Z.L. and 81302293 to F.S.].

\section{Author details}

${ }^{1}$ Department of Epidemiology and Biostatistics, , Tianjin Medical University Cancer Institute and Hospital, Tianjin 300060, P. R. China. ${ }^{2}$ Department of Biochemistry and Molecular Biology, Tianjin Medical University Cancer Institute and Hospital, Tianjin 300060, P. R. China. ${ }^{3}$ Department of Breast Surgery, Tianjin Medical University Cancer Institute and Hospital, Tianjin 300060, P. R. China. ${ }^{4}$ Key Laboratory of Breast Cancer Prevention and Therapy, Ministry of Education, Key Laboratory of Cancer Prevention and Therapy, Tianjin, National Clinical Research Center of Cancer, Tianjin Medical University Cancer Institute and Hospital, Tianjin 300060, P. R. China. ${ }^{5}$ The Institute for Chemical Carcinogenesis, State Key Lab of Respiratory Disease, Guangzhou Medical University, Guangzhou 510182, China.

Received: 13 October 2014 Accepted: 17 July 2015

Published online: 30 July 2015 


\section{References}

1. Vousden $\mathrm{KH}$, Prives C. Blinded by the Light: The Growing Complexity of p53. Cell. 2009;137:413-31.

2. Bunz F, Dutriaux A, Lengauer C, Waldman T, Zhou S, Brown JP, et al. Requirement for $p 53$ and $p 21$ to sustain $\mathrm{G} 2$ arrest after DNA damage. Science. 1998:282:1497-501.

3. Hu W, Feng Z, Teresky AK, Levine AJ. p53 regulates maternal reproduction through LIF. Nature. 2007:450:721-4.

4. Lowe CR, MacMahon B. Breast cancer and reproduction. Lancet. 1970;2:1137.

5. Yuan JM, Yu MC, Ross RK, Gao YT, Henderson BE. Risk factors for breast cancer in Chinese women in Shanghai. Cancer Res. 1988;48:1949-53.

6. Bernstein L. Epidemiology of endocrine-related risk factors for breast cancer. J Mammary Gland Biol Neoplasia. 2002;7:3-15

7. Albrektsen G, Heuch I, Thoresen S, Kvåle G. Family history of breast cancer and short-term effects of childbirths on breast cancer risk. Int J Cancer. 2006:119:1468-74.

8. Bruzzi P, Negri E, La Vecchia C, Decarli A, Palli D, Parazzini F, et al. Short term increase in risk of breast cancer after full term pregnancy. Br Med J. 1988:297:1096-8.

9. Russo J, Moral R, Balogh GA, Mailo D, Russo $\mathrm{IH}$. The protective role of pregnancy in breast cancer. Breast Cancer Res. 2005;7:131-42.

10. Li Z, Zhang Q, Mao JH, Weise A, Mrasek K, Fan X, et al. An HDAC1-binding domain within FATS bridges p21 turnover to radiation-induced tumorigenesis. Oncogene. 2010;29:2659-71.

11. Zhang X, Zhang Q, Zhang J, Qiu L, Yan SS, Feng J, et al. FATS is a transcriptional target of p53 and associated with antitumor activity. Mol Cancer. 2010:9:244.

12. Yan S, Qiu L, Ma K, Zhang X, Zhao Y, Zhang J, Li X, Hao X, Li Z. FATS is an E2-independent ubiquitin ligase that stabilizes p53 and promotes its activation in response to DNA damage. Oncogene 2013; e-pub ahead of print 18 November 2013; doi: 10.1038/onc.2013.494

13. Ma K, Qiu L, Mrasek K, Zhang J, Liehr T, Quintana LG, et al. Common fragile sites: genomic hotspots of DNA damage and carcinogenesis. Int J Mol Sci. 2012:13:11974-99.

14. Zhang J, Gu L, Zhao L, Zhang X, Qiu L, Li Z. Expression level of novel tumor suppressor gene FATS is associated with the outcome of node positive breast cancer. Chin Med J. 2011;124:2894-8.

15. Tian Y, Zhang J, Yan S, Qiu L, Li Z. FATS expression is associated with cisplatin sensitivity in non small cell lung cancer. Lung Cancer. 2012;76:416-22.

16. Song $F$, Ji $P$, Zheng $H$, Song F, Wang $Y$, Hao $X$, et al. Definition of a functional single nucleotide polymorphism in the cell migration inhibitory gene MIIP that affects the risk of breast cancer. Cancer Res. 2010:70:1024-32.

17. Masters JR, Thomson JA, Daly-Burns B, Reid YA, Dirks WG, Packer P, et al Short tandem repeat profiling provides an international reference standard for human cell lines. Proc Natl Acad Sci U S A. 2001;98:8012-7.

18. DerSimonian R, Laird N. Meta-analysis in clinical trials. Control Clin Trials. 1986;7:177-88.

19. Mantel N, Haenszel W. Statistical aspects of the analysis of data from retrospective studies of disease. J Natl Cancer Inst. 1959;22:719-48.

20. Albrektsen G, Heuch I, Thoresen S, Kvåle G. Clinical stage of breast cancer by parity, age at birth, and time since birth: a progressive effect of pregnancy hormones? Cancer Epidemiol Biomarkers Prev. 2006;15:65-9.

21. Levine AJ, Tomasini R, McKeon FD, Mak TW, Melino G. The p53 family: guardians of maternal reproduction. Nat Rev Mol Cell Biol. 2011;12:259-65.

22. Schmidt MK, Reincke S, Broeks A, Braaf LM, Hogervorst FB, Tollenaar RA, et al. Do MDM2 SNP309 and TP53 R72P interact in breast cancer susceptibility? A large pooled series from the breast cancer association consortium. Cancer Res. 2007:67:9584-90.

23. Baynes C, Healey CS, Pooley KA, Scollen S, Luben RN, Thompson DJ, et al. Common variants in the ATM, BRCA1, BRCA2, CHEK2 and TP53 cancer susceptibility genes are unlikely to increase breast cancer risk. Breast Cancer Res. 2007:9:R27.

\section{Submit your next manuscript to BioMed Central and take full advantage of:}

- Convenient online submission

- Thorough peer review

- No space constraints or color figure charges

- Immediate publication on acceptance

- Inclusion in PubMed, CAS, Scopus and Google Scholar

- Research which is freely available for redistribution

Submit your manuscript at www.biomedcentral.com/submit 